\title{
Obesity and lipid-related parameters for predicting metabolic syndrome in Chinese elderly population
}

\author{
Zhan $\mathrm{Gu}^{1 \dagger}$, Ping Zhu ${ }^{2 \dagger}$, Qiao Wang ${ }^{1}$, Huayu He${ }^{1}$, Jingjuan Xu' ${ }^{1}$ Li Zhang ${ }^{1}$, Dong $\mathrm{Li}^{3}$, Jianying Wang ${ }^{1}$, Xiaojuan Hu${ }^{1}$,
} Guang $\mathrm{Ji}^{1,4}$, Lei Zhang ${ }^{1 *}$ and Baocheng Liu ${ }^{1 *}$

\begin{abstract}
Background: The present study evaluated the predictive ability of five known "best" obesity and lipid-related parameters, including body mass index (BMI), waist-to-height ratio (WHtR), triglyceride-to-high-density-lipoproteincholesterol (TG/HDL-C), lipid accumulation product (LAP) and visceral adiposity index (VAl), in identifying metabolic syndrome (MetS) in Chinese elderly population.

Methods: A total of 6722 elderly Chinese subjects ( $\geq 60$ years) were recruited into our community-based crosssectional study from April 2015 to July 2017. The anthropometrics, blood pressure, fasting plasma glucose, blood lipid profiles, family history and health-related behaviours were assessed.
\end{abstract}

Results: The prevalence of MetS was $40.4 \%$ (32.5\% in males and $47.2 \%$ in females). With the increase in the number of MetS components (from 0 to 5), all the five parameters showed an increase trend in both genders (all $P$ for trend $<0.001)$. According to receiver operating characteristic curve $(\mathrm{ROC})$ analyses, all the five parameters performed high predictive value in identifying MetS. The statistical significance of the areas under the curves (AUCS) differences suggested that the AUCs of LAP were the greatest among others in both genders (AUCs were 0.897 in males and 0.875 in females). The optimal cut-off values of LAP were 26.35 in males and 31.04 in females. After adjustment for potentially confounding factors, LAP was strongly associated with the odds of having MetS in both genders, and ORs for MetS increased across quartiles using multivariate logistic regression analysis $(P<0.001)$.

Conclusion: LAP appeared to be a superior parameter for predicting MetS in both Chinese elderly males and females, better than VAl, TG/HDL-C, WHtR and BMI.

Keywords: Metabolic syndrome, Obesity and lipid-related parameters, Chinese elderly population, Lipid accumulation product, Visceral adiposity index, Triglyceride-to-high-density-lipoprotein-cholesterol, Waist-to-height ratio, Body mass index

\section{Background}

Metabolic syndrome (MetS) is a complex of interrelated risk factors for cardiovascular disease (CVD) and diabetes. These factors include central obesity, elevated blood pressure (BP), elevated fasting plasma glucose (FPG) levels, elevated triglyceride (TG) levels, and reduced high-density lipoprotein cholesterol (HDL-C)

\footnotetext{
* Correspondence: zhanglei37@sina.com; baochliumed@126.com

'Zhan Gu and Ping Zhu contributed equally to this work.

'Shanghai Innovation Center of TCM Health Service, Shanghai University of

Traditional Chinese Medicine, Shanghai 201203, China

Full list of author information is available at the end of the article
}

levels [1-3]. As a result of rapid changes in lifestyle and dietary habits, MetS has recently become an epidemic threatening the health of Chinese people, especially those elderly ones. A systematic review has shown that the prevalence of MetS is $24.5 \%$ in China, and it is higher (32.4\%) in Chinese elderly [4]. Additionally, a national study in 2014-2015 found that the prevalence of MetS was higher in the Chinese elderly and females [5]. Hence, early identification of subjects at high risk of MetS is imperative to prevent occurence and progression of MetS.

(c) The Author(s). 2018 Open Access This article is distributed under the terms of the Creative Commons Attribution 4.0 International License (http://creativecommons.org/licenses/by/4.0/), which permits unrestricted use, distribution, and 
Obesity and blood lipids are closely related to CVD and diabetes. Various obesity and lipid-related parameters, especially body mass index (BMI), waist-to-height ratio (WHtR), triglyceride-to-high-density-lipoproteincholesterol (TG/HDL-C), lipid accumulation product (LAP) and visceral adiposity index (VAI), have commonly been used to predict MetS and each one of these five parameters has ever been reported as the "best" parameter for predicting MetS in previous studies [6-12]. Nevertheless, there has been no study on all these five known "best" parameters for predicting MetS to date via PubMed database (https://www.ncbi.nlm.nih.gov/pubmed) and it has still been argued about which parameter conveys the highest risk of MetS.

BMI is a measurement of body fat based on height and weight [13]. WHtR reflects abdominal or central obesity. BMI and WHtR have been regarded as two powerful anthropometric parameters for detection of cardiometabolic risk factors in previous studies $[6,7,14$, 15]. Evidence has shown that lipid ratios perform better than individual lipids in predicting cardiometabolic risk and TG/HDL-C is considered to be a better marker to identify MetS $[8,9]$. LAP and VAI are two recent indicators and are used to estimate visceral obesity. LAP is calculated as a combination of waist circumference (WC) and TG [16]. VAI, introduced by the AlkaMeSy Study Group, comprises anthropometric parameters (BMI and WC) and lipid parameters (TG and HDL-C) [17]. LAP and VAI have been proposed as simple and useful markers of MetS in recent studies [10-12].

It is important to identify a parameter to serve as a quick and powerful tool for prevention and simple diagnosis of MetS in Chinese elderly population. Therefore, the purpose of our study is to compare the predictive ability of these five "best" obesity and lipid-related parameters for identifying males and females with MetS in elderly Chinese subjects.

\section{Methods}

\section{Study subjects}

This study is a community-based cross-sectional investigation for the elderly population in Shanghai, China. A total of 7098 residents from Zhangiiang community of Shanghai aged $\geq 60$ years were recruited into our study from April 2015 to July 2017. The study was performed according to the guidelines of the Helsinki Declaration. A standard protocol was designed by Shanghai innovation center of TCM health service and was approved by the Ethics Committee of Shanghai University of Traditional Chinese Medicine. Written informed consent was obtained from all subjects.

The inclusion criteria included age $\geq 60$ years, local residents in Shanghai, complete data measurements and informed consents. Subjects with mental disorders, malignant tumors or incomplete recorded information were excluded from this project based on their medical records. After investigation, 376 subjects were excluded from the study. A total of 6722 Chinese elderly subjects (3077 males and 3645 females) with complete data were finally included in this study.

\section{Data measurements}

The obesity and lipid-related parameters included BMI, WHtR, TG/HDL-C, LAP and VAI. Height and weight were measured to the nearest $0.1 \mathrm{~cm}$ and $0.1 \mathrm{~kg}$ using electronic measurement instrument (Shengyuan; Zhengzhou, China). WC was measured to the nearest $0.1 \mathrm{~cm}$ using a flexible metric measuring tape (Pudong CDC; Shanghai, China). All subjects were measured wearing light clothing without hats and shoes. Blood pressure was measured with electronic sphygmomanometers (Biospace; Cheonan, South Korea) using the standard recommended procedures. Blood samples were obtained from the antecubital vein in the morning after an overnight fasting period. FPG, TG, HDL-C, low-density lipoprotein cholesterol (LDL-C) and total cholesterol (TC) were measured using an automatic biochemistry analyzer (Hitachi; Tokyo, Japan). Current smoking (current smokers or non-smokers), alcohol consumption (currently consume alcohol or do not consume alcohol) and family history of CVD (yes or no) were recorded by self-report.

BMI was calculated as bodyweight $(\mathrm{kg}) /$ height $^{2}\left(\mathrm{~m}^{2}\right)$. WHtR was calculated as WC $(\mathrm{cm}) /$ height $(\mathrm{cm})$. TG/HDL-C was calculated as TG $(\mathrm{mmol} / \mathrm{L}) / \mathrm{HDL}-\mathrm{C}(\mathrm{mmol} / \mathrm{L})$. LAP and VAI were calculated based on the gender-specific mathematical model formula. LAP $=[\mathrm{WC}(\mathrm{cm})-65] \times \mathrm{TG}(\mathrm{mmol} / \mathrm{L})$ for males, and [WC $(\mathrm{cm})-58] \times \mathrm{TG}(\mathrm{mmol} / \mathrm{L})$ for females [16]. WC values $\leq 65 \mathrm{~cm}$ in males and $\leq 58 \mathrm{~cm}$ in females were revised upward to $66.0 \mathrm{~cm}$ and $59.0 \mathrm{~cm}$ to avoid invalid data $[16] . \quad \mathrm{VAI}=[\mathrm{WC} \quad(\mathrm{cm}) /[39.68+(1.88 \times \mathrm{BMI})]] \times[\mathrm{TG}$ $(\mathrm{mmol} / \mathrm{L}) / 1.03] \times[1.31 / \mathrm{HDL}-\mathrm{C}(\mathrm{mmol} / \mathrm{L})]$ for males, and $[\mathrm{WC}(\mathrm{cm}) /[36.58+(1.89 \times \mathrm{BMI})]] \times[\mathrm{TG}(\mathrm{mmol} / \mathrm{L}) /$ $0.81] \times[1.52 / \mathrm{HDL}-\mathrm{C}(\mathrm{mmol} / \mathrm{L})]$ for females [17] .

\section{Definition of MetS}

According to the diagnosis criteria proposed by the International Diabetes Federation (IDF) and the American Heart Association (AHA)/National Heart, Lung and Blood Institute (NHLBI) in 2009 [1] combined with an amended definition of abdominal obesity for Chinese population [18], MetS was defined as the presence of three or more of those following features: (a) central obesity: WC $\geq 90 \mathrm{~cm}$ for males or $\geq 85 \mathrm{~cm}$ for females; (b) elevated BP: systolic blood pressure (SBP) $\geq 130$ $\mathrm{mmHg}$ or diastolic blood pressure (DBP) $\geq 85 \mathrm{mmHg}$, or ongoing antihypertensive medications; (c) elevated FPG: FPG $\geq 5.6 \mathrm{mmol} / \mathrm{L}$, or ongoing anti-diabetic treatment; 
(d) elevated TG: TG $\geq 1.7 \mathrm{mmol} / \mathrm{L}$; and (e) reduced HDL-C: HDL-C $<1.0 \mathrm{mmol} / \mathrm{L}$ in males and $<1.3 \mathrm{mmol} / \mathrm{L}$ in females.

\section{Statistical analyses}

All of the descriptive statistics for all of the variables were calculated. Categorical variables were expressed as counts or percentages and compared using Pearson's $X^{2}$ tests. Continuous variables were expressed as mean \pm standard deviation and compared using two-sided $t$ tests. One-way ANOVA test was used for comparisons of the levels of obesity and lipid-related parameters across number of MetS components. To compare the predictive ability and determine the optimal cut-off values of the parameters for predicting MetS, receiver operating characteristic curve (ROC) analyses were used. The areas under the receiver operating characteristic curves (AUCs) were calculated, and the optimal cut-off values were identified from the maximum Youden index (sensitivity plus specificity - 1) to determine the appropriate parameters. Multivariate logistic regression analysis was applied to calculate odds ratios (ORs) and 95\% confidence intervals (CIs) for MetS across quartiles of the parameters, with quartile 1 as reference group, adjusting for potentially confounding factors such as age, current smoking, alcohol consumption and family history of CVD. Considering significant gender differences in body fat distribution and MetS [19, 20], all statistical analyses were separately performed within males and females.

$P$ values $<0.05$ were set as significant for all of the statistical tests for bilateral contrasts. All statistical analyses were conducted using SPSS version 21.0 (SPSS; Chicago, USA). The statistical significance of the differences in the AUCs was analyzed using MedCalc version 17.1.0 (MedCalc; Ostend, Belgium) with the algorithm developed by DeLong's research team [21].

\section{Results}

\section{Baseline characteristics}

As shown in Table 1, the subjects with MetS tended to have significantly higher BMI, WC, WHtR, SBP, DBP, FPG, TG, TG/HDL-C, LAP and VAI, and lower HDL-C compared with the subjects without MetS in both genders $(P<0.001)$. The subjects with MetS had higher prevalence of current smoking and alcohol consumption compared with the subjects without MetS in both genders $(P<0.05)$. Age, LDL-C and the prevalence of family history of CVD were not significantly different between males with MetS and without MetS. TC was higher in males with MetS compared to without MetS $(P<0.05)$. Age and the prevalence of family history of CVD were higher in females with MetS compared to without MetS

Table 1 The baseline characteristics of the study subjects

\begin{tabular}{|c|c|c|c|c|c|c|}
\hline \multirow[t]{2}{*}{ Variable } & \multicolumn{3}{|l|}{ Male $(n=3077)$} & \multicolumn{3}{|l|}{ Female $(n=3645)$} \\
\hline & With MetS $(n=999)$ & Without MetS $(n=2078)$ & $P$ value & With MetS $(n=1719)$ & Without MetS $(n=1926)$ & $P$ value \\
\hline Age (years) & $70.02 \pm 7.21$ & $69.98 \pm 7.42$ & 0.885 & $70.59 \pm 7.51$ & $69.70 \pm 7.75$ & $<0.001$ \\
\hline Current smoking & $94(9.41 \%)$ & $129(6.21 \%)$ & 0.001 & $17(0.99 \%)$ & $5(0.26 \%)$ & 0.005 \\
\hline Alcohol consumption & $260(26.03 \%)$ & $242(11.65 \%)$ & $<0.001$ & $172(10.01 \%)$ & $75(3.89 \%)$ & $<0.001$ \\
\hline Family history of CVD & $249(24.92 \%)$ & $513(24.69 \%)$ & 0.886 & $535(31.12 \%)$ & $423(21.96 \%)$ & $<0.001$ \\
\hline BMI $\left(\mathrm{kg} / \mathrm{m}^{2}\right)$ & $26.02 \pm 2.83$ & $23.14 \pm 3.05$ & $<0.001$ & $25.74 \pm 3.77$ & $22.58 \pm 3.04$ & $<0.001$ \\
\hline WC (cm) & $90.6 \pm 7.8$ & $81.2 \pm 7.8$ & $<0.001$ & $85.8 \pm 8.6$ & $76.7 \pm 7.9$ & $<0.001$ \\
\hline $\mathrm{WHtR}$ & $0.54 \pm 0.05$ & $0.49 \pm 0.05$ & $<0.001$ & $0.55 \pm 0.06$ & $0.50 \pm 0.05$ & $<0.001$ \\
\hline $\mathrm{SBP}(\mathrm{mmHg})$ & $149(136,162)$ & $136(122,153)$ & $<0.001$ & $148(136,161)$ & $134(120,151)$ & $<0.001$ \\
\hline $\mathrm{DBP}(\mathrm{mmHg})$ & $84.6 \pm 11.5$ & $79.7 \pm 12.3$ & $<0.001$ & $84.9 \pm 11.2$ & $79.8 \pm 12.1$ & $<0.001$ \\
\hline FPG (mmol/L) & $6.3(5.7,7.4)$ & $5.5(5.1,6.0)$ & $<0.001$ & $6.0(5.6,6.9)$ & $5.4(5.1,5.8)$ & $<0.001$ \\
\hline TC $(\mathrm{mmol} / \mathrm{L})$ & $4.81(4.21,5.45)$ & $4.72(4.16,5.33)$ & 0.016 & $5.27(4.64,5.91)$ & $5.36(4.75,5.94)$ & 0.003 \\
\hline TG $(\mathrm{mmol} / \mathrm{L})$ & $2.11 \pm 1.57$ & $1.09 \pm 0.56$ & $<0.001$ & $2.03 \pm 1.35$ & $1.15 \pm 0.49$ & $<0.001$ \\
\hline $\mathrm{HDL}-\mathrm{C}(\mathrm{mmol} / \mathrm{L})$ & $1.02 \pm 0.20$ & $1.25 \pm 0.25$ & $<0.001$ & $1.17 \pm 0.20$ & $1.46 \pm 0.27$ & $<0.001$ \\
\hline LDL-C (mmol/L) & $3.01(2.47,3.56)$ & $3.00(2.47,3.54)$ & 0.795 & $3.24(2.66,3.85)$ & $3.37(2.77,3.89)$ & 0.005 \\
\hline $\mathrm{TG} / \mathrm{HDL}-\mathrm{C}$ & $2.20 \pm 2.07$ & $0.93 \pm 0.60$ & $<0.001$ & $1.88 \pm 1.65$ & $0.84 \pm 0.48$ & $<0.001$ \\
\hline LAP & $51.71 \pm 38.60$ & $18.27 \pm 13.53$ & $<0.001$ & $55.25 \pm 40.15$ & $21.75 \pm 13.16$ & $<0.001$ \\
\hline VAl & $2.84 \pm 2.61$ & $1.16 \pm 0.75$ & $<0.001$ & $3.54 \pm 3.16$ & $1.52 \pm 0.88$ & $<0.001$ \\
\hline
\end{tabular}

Data are expressed as mean \pm standard deviation, median (interquartile range $25-75 \%$ ), or counts (percentages)

MetS metabolic syndrome, CVD cardiovascular disease, BMI body mass index, WC waist circumference, WHtR waist-to-height ratio, SBP systolic blood pressure, DBP diastolic blood pressure, FPG fasting plasma glucose, $T C$ total cholesterol, $T G$ triglyceride, $H D L-C$ high-density lipoprotein cholesterol, $L D L-C$ low-density lipoprotein cholesterol, LAP lipid accumulation product, VAI visceral adiposity index 


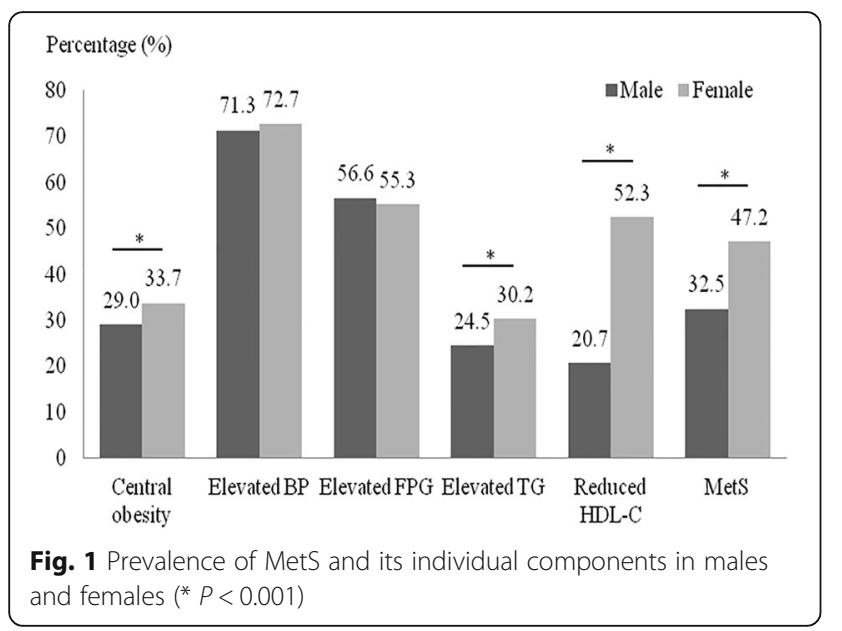

$(P<0.001)$. TC and LDL-C were higher in females without MetS compared to with MetS $(P<0.05)$.

\section{Prevalence of MetS}

Prevalence of MetS and its individual components in Chinese elderly males and females are shown in Fig. 1. Approximately $40.4 \%$ of the subjects had MetS $(32.5 \%$ in males and $47.2 \%$ in females). The prevalence of MetS in females was significantly higher than that in males $(P<0.001)$. Among the individual components of MetS, the prevalence of central obesity, elevated TG and reduced HDL-C in females were significantly higher than that in males $(P<0.001)$. The prevalence of elevated BP and elevated FPG were not significantly different between males and females.

\section{Levels of the parameters across number of MetS components}

Levels of the five obesity and lipid-related parameters in the study subjects across number of MetS components are listed in Table 2. With the increase in the number of MetS components (from 0 to 5), BMI, WHtR, TG/HDL-C, LAP and VAI all showed an increase trend in both genders (all $P$ for trend $<0.001$ ).

\section{The parameters for predicting MetS}

ROC curves for the five parameters to predict MetS in males and females are shown in Fig. 2. The AUCs, optimal cut-off values, sensitivity, specificity and Youden index of the five parameters for predicting MetS are shown in Table 3. The statistical significance of the AUCs differences of the five parameters in males suggested that the AUC of LAP (AUC: 0.897, 95\% CI: 0.885-0.907) was the greatest among others, followed by VAI, TG/HDL-C, WHtR and BMI. In females, the AUC of LAP (AUC: $0.875,95 \%$ CI: 0.864-0.886) was also the greatest, followed by VAI, TG/HDL-C, WHtR and BMI (WHtR and BMI did not differ). LAP had the strongest predictive ability for both genders. The optimal cut-off values of LAP were 26.35 in males and 31.04 in females.

\section{MetS risk across quartiles of LAP}

The adjusted ORs and 95\%CI of MetS across quartiles of LAP are shown in Table 4. After adjustment for age, current smoking, alcohol consumption and family history of CVD, LAP was strongly associated with the odds of having MetS in both genders, and ORs for MetS increased across quartiles $(P<0.001)$. The study subjects in the highest quartiles of LAP had a 216.630-fold increased risk in males and a 114.291-fold increased risk in females of suffering from MetS compared with those in the reference group.

\section{Discussion}

In this study, we have investigated the predictive value of BMI, WHtR, TG/HDL-C, LAP and VAI, five known "best" obesity and lipid-related parameters, in identifying MetS in the Chinese elderly. The results showed that

Table $\mathbf{2}$ Levels of the five parameters across number of MetS components

\begin{tabular}{|c|c|c|c|c|c|c|c|}
\hline Variable & 0 & 1 & 2 & 3 & 4 & 5 & $P$ for trend \\
\hline Male & $(n=307)$ & $(n=796)$ & $(n=975)$ & $(n=611)$ & $(n=298)$ & $(n=90)$ & \\
\hline BMl & $21.77 \pm 2.60$ & $22.66 \pm 3.11$ & $23.97 \pm 2.89$ & $25.68 \pm 2.84$ & $26.26 \pm 2.69$ & $27.55 \pm 2.61$ & $<0.001$ \\
\hline WHtR & $0.47 \pm 0.04$ & $0.48 \pm 0.04$ & $0.50 \pm 0.05$ & $0.54 \pm 0.05$ & $0.55 \pm 0.04$ & $0.57 \pm 0.04$ & $<0.001$ \\
\hline TG/HDL-C & $0.74 \pm 0.30$ & $0.85 \pm 0.43$ & $1.05 \pm 0.74$ & $1.70 \pm 1.19$ & $2.65 \pm 1.97$ & $4.07 \pm 3.40$ & $<0.001$ \\
\hline LAP & $11.86 \pm 8.32$ & $15.70 \pm 10.87$ & $22.39 \pm 15.39$ & $39.76 \pm 20.32$ & $62.16 \pm 34.16$ & $98.27 \pm 80.30$ & $<0.001$ \\
\hline VAl & $0.91 \pm 0.38$ & $1.05 \pm 0.54$ & $1.32 \pm 0.93$ & $2.17 \pm 1.41$ & $3.44 \pm 2.47$ & $5.36 \pm 4.62$ & $<0.001$ \\
\hline Female & $(n=256)$ & $(n=676)$ & $(n=994)$ & $(n=862)$ & $(n=631)$ & $(n=226)$ & \\
\hline BMI & $21.15 \pm 2.62$ & $21.95 \pm 2.87$ & $23.37 \pm 3.02$ & $25.07 \pm 3.93$ & $25.98 \pm 3.50$ & $27.61 \pm 3.15$ & $<0.001$ \\
\hline WHtR & $0.47 \pm 0.05$ & $0.49 \pm 0.05$ & $0.51 \pm 0.06$ & $0.54 \pm 0.06$ & $0.56 \pm 0.06$ & $0.59 \pm 0.04$ & $<0.001$ \\
\hline TG/HDL-C & $0.61 \pm 0.24$ & $0.74 \pm 0.32$ & $0.96 \pm 0.57$ & $1.44 \pm 1.23$ & $2.11 \pm 1.60$ & $2.91 \pm 2.40$ & $<0.001$ \\
\hline LAP & $14.14 \pm 8.25$ & $18.38 \pm 10.07$ & $26.00 \pm 14.44$ & $39.68 \pm 20.38$ & $61.59 \pm 38.48$ & $96.96 \pm 61.52$ & $<0.001$ \\
\hline VAl & $1.09 \pm 0.43$ & $1.33 \pm 0.60$ & $1.76 \pm 1.03$ & $2.65 \pm 2.17$ & $3.97 \pm 3.00$ & $5.71 \pm 4.97$ & $<0.001$ \\
\hline
\end{tabular}




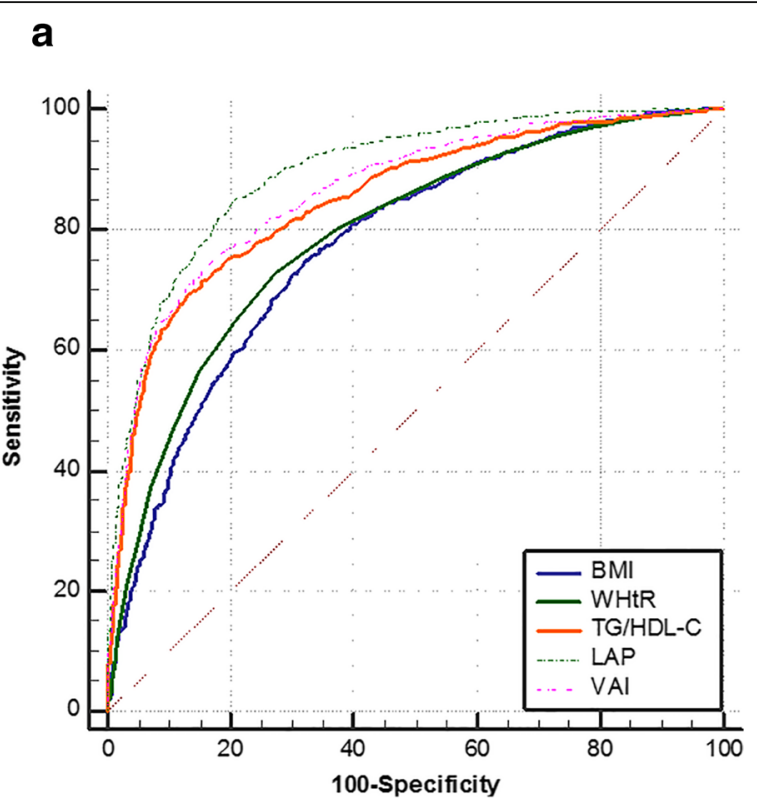

b

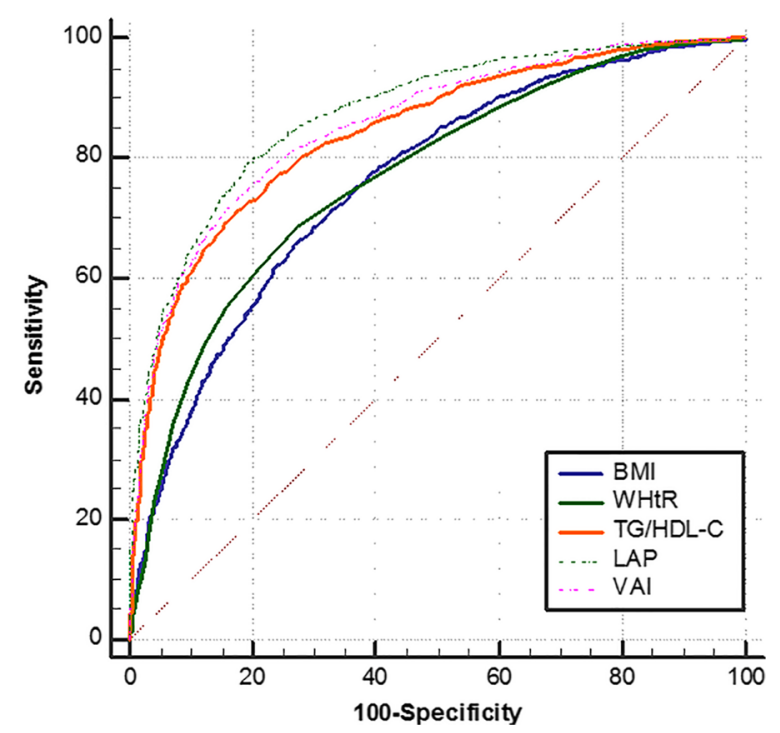

Fig. 2 ROC curves for the five parameters to predict MetS in males (a) and females (b)

LAP performed better than the other four parameters for predicting MetS in both elderly males and females. Additionally we also confirmed LAP was strongly associated with MetS in both genders. To the best of our knowledge, this is the first comprehensive study to evaluate the predictive ability of these five "best" obesity and lipid-related parameters with MetS and to focus on a large sample of Chinese elderly population.

The prevalence of MetS is high and increasing rapidly in Chinese elderly population. A cross-sectional survey in a representative sample of elderly people in Beijing showed $58.1 \%$ of the participants had MetS and MetS was more common in females than males [22]. We found the prevalence of MetS was $40.4 \%$, with an obviously higher prevalence was shown in females as well. As a novel marker of excessive lipid accumulation, the LAP introduced by Kahn [16] has been widely applied for recognizing cardiovascular risk over the past decade. Our study found LAP had the strongest predictive ability for identifying MetS in both genders, in line with many previous studies [10, 12, 23-27]. A recent study of 992 Chinese middle-aged and elderly subjects showed that LAP performed better than VAI and the product of triglycerides and glucose (TyG) in identifying MetS [10]. And the optimal cut-off value of LAP was 31.465 for the elderly, greater than our findings. Guo et al. suggested LAP was a better indicator for predicting MetS compared to VAI, body adiposity index (BAI) and WHtR in low income rural adults of Xinjiang, China [12]. The results showed the cut-off values of LAP were 34.7 (AUC: 0.853 ) in males and 27.3 (AUC: 0.817 ) in females. One study from Brazil also suggested that LAP had better predictive ability for MetS in the elderly and the cut-off value was 32.3 (AUC: 0.849) [28]. Our results validated the predictive value of LAP in the Chinese elderly. In addition, the strong ability of LAP for predicting MetS were also identified in Spanish, Argentinian and Iranian populations [23, 25, 27]. We also confirmed the increase of MetS risk across quartiles of LAP, consistent with both Guo et al.'s research and Ma et al.'s research [12, 29]. Moreover, LAP was also found to be a strong predictor of metabolic and related disorders, such as diabetes, insulin resistance and non-alcoholic fatty liver disease [30-32]. Indeed, LAP is a simple and quick parameter, and it only requires the WC measurement and serum TG testing. Therefore, LAP is expected to be a powerful tool and the "best" parameter among multiple obesity and lipid-related parameters to identify Chinese elderly subjects at high risk of MetS in clinical practice.

In our present study, VAI and TG/HDL-C were defined as "excellent" parameters $(0.8 \leq \mathrm{AUC}<0.9)$ based on Hosmer and Lemeshow's criteria, while WHtR and BMI were "acceptable" parameters $(0.7 \leq \mathrm{AUC}<0.8)$. The VAI, as a surrogate marker of adipose tissue function and distribution, was independently correlated with cardiometabolic risk [17, 33, 34]. Li et al.'s research and Guo et al.'s research both suggested VAI could be applied for identifying MetS in Chinese population [10, 12], supporting our findings. One study from Iran also confirmed the predictive value of VAI was high in identifying MetS [35]. The TG/HDL-C has been proposed as a potential tool to identify patients at increased risk of MetS $[8,9,36,37]$. A study from Canada indicated that TG/ HDL-C was a superior marker to identify MetS compared 
Table 3 The AUCS, optimal cut-off values, sensitivity, specificity and Youden index of the five parameters for predicting MetS

\begin{tabular}{|c|c|c|c|c|c|}
\hline Variable & $\mathrm{AUC}(95 \% \mathrm{Cl})$ & Cut-off value & Sensitivity (\%) & Specificity (\%) & Youden index (\%) \\
\hline \multicolumn{6}{|l|}{ Male } \\
\hline BMI & $0.775(0.760-0.789)$ & 24.17 & 74.97 & 67.81 & 42.78 \\
\hline $\mathrm{WHtR}$ & $0.791(0.776-0.805)$ & 0.51 & 72.87 & 72.52 & 45.39 \\
\hline TG/HDL-C & $0.851(0.838-0.864)$ & 1.38 & 69.47 & 86.72 & 56.19 \\
\hline LAP & $0.897(0.885-0.907)$ & 26.35 & 85.09 & 79.31 & 64.39 \\
\hline VAl & $0.865(0.853-0.877)$ & 1.63 & 74.17 & 83.64 & 57.81 \\
\hline \multicolumn{6}{|l|}{ Female } \\
\hline BMI & $0.760(0.746-0.774)$ & 24.03 & 66.67 & 72.17 & 38.84 \\
\hline $\mathrm{WH} t \mathrm{R}$ & $0.769(0.755-0.782)$ & 0.52 & 68.64 & 72.53 & 41.18 \\
\hline $\mathrm{TG} / \mathrm{HDL}-\mathrm{C}$ & $0.843(0.831-0.855)$ & 1.13 & 71.09 & 82.76 & 53.85 \\
\hline LAP & $0.875(0.864-0.886)$ & 31.04 & 79.17 & 80.69 & 59.86 \\
\hline VAl & $0.856(0.844-0.867)$ & 2.05 & 73.82 & 81.98 & 55.81 \\
\hline
\end{tabular}

to TC/HDL-C, LDL-C/HDL-C, and nonHDL-C/HDL-C based on a multiethnic sample [8]. Chen et al. proposed TG/HDL-C was a better predictor of MetS in Chinese Uighur females [9]. Consistent with these results, we also found VAI and TG/HDL-C had good predictive accuracy to identify MetS in the Chinese elderly. WHtR and BMI are two common anthropometric parameters and have been applied for detection of cardiometabolic risk for a long time. In our previous study, WHtR and BMI could be used for prediction of multiple metabolic risk factors in the Chinese elderly [14]. Yang et al. indicated WHtR was better than BMI and WC as a screening tool for MetS [7]. And Wang et al.'s research suggested BMI was useful for predicting MetS [6]. Our results found WHtR and BMI were both acceptable parameters for predicting MetS, but inferior to LAP, VAI and TG/HDL-C. Of note, our study focuses on the Chinese elderly aged 60 years and above in Shanghai, and the differences in the results with other

Table 4 The MetS risk across quartiles of LAP

\begin{tabular}{clc}
\hline & OR $(95 \% \mathrm{Cl})$ & $P$ value \\
\hline Male & & \\
Q1 & Reference & $<0.001$ \\
Q2 & $4.908(2.824-8.531)$ & $<0.001$ \\
Q3 & $29.791(17.686-50.179)$ & $<0.001$ \\
Q4 & $216.630(127.064-369.328)$ & \\
Female & & $<0.001$ \\
Q1 & Reference & $<0.001$ \\
Q2 & $4.185(3.169-5.528)$ & $<0.001$ \\
Q3 & $17.344(13.203-22.785)$ & \\
Q4 & $114.291(82.115-159.075)$ & \\
\hline
\end{tabular}

Multivariate logistic regression analysis, adjustment for age, current smoking, alcohol consumption and family history of CVD findings may be due to the age and regional distributional differences of the samples.

There are, however, several limitations of this study. The subjects in this study were all from Shanghai, and the sample may not fully reflect all Chinese elderly. This study included quite a large number of Chinese elderly, but we only enrolled subjects who had completed the comprehensive health check study, which may have biased our primary findings. Moreover, our analyses were adjusted for age, current smoking, alcohol consumption and family history of CVD, but not for physical activity and diet known to influence obesity and lipids. Further studies with multi centers, a larger sample size and more detailed information collection are needed to assess the predictive value of obesity and lipid-related parameters in identifying elderly subjects at high risk of MetS.

\section{Conclusion}

In conclusion, our study performed the predictive ability of obesity and lipid-related parameters, including BMI, WHtR, TG/HDL-C, LAP and VAI, in identifying MetS in Chinese elderly population. LAP appeared to be a superior parameter for predicting MetS in both Chinese elderly males and females, and was strongly associated with MetS in both genders. Namely, LAP readings of 26.35 or greater in males and 31.04 or greater in females can help to identify the elderly subjects at high risk of MetS.

\section{Abbreviations}

AUC: Area under the receiver operating characteristic curve; BMI: Body mass index; CVD: Cardiovascular disease; DBP: Diastolic blood pressure; FPG: Fasting plasma glucose; HDL-C: High-density lipoprotein cholesterol; LAP: Lipid accumulation product; MetS: Metabolic syndrome;

ROC: Receiver operating characteristic curve; SBP: Systolic blood pressure; TG: Triglyceride; VAI: Visceral adiposity index; WC: Waist circumference; WHtR: Waist-to-height ratio 


\section{Acknowledgements}

We thank all the partners and staff who helped us in the process of this study.

\section{Funding}

This work was supported by the National Natural Science Foundation of China (No. 816220108030, No. 81603411, No. 81573814) and China Postdoctoral Science Foundation (No. 2018 M630465).

\section{Availability of data and materials}

The datasets used and/or analyzed during the current study are available from the corresponding authors upon reasonable request.

\section{Authors' contributions}

ZG, LZ and BL designed the study; ZG, PZ, QW, HH, JX, LZ and DL colleted the data; $Z G, P Z$, JW and $X H$ analyzed the data; $Z G$ and $P Z$ wrote the manuscript; GJ, LZ and BL revised the manuscript content. All authors have read and approved the final submitted manuscript

\section{Ethics approval and consent to participate}

This study was approved by the Ethics Committee of Shanghai University of Traditional Chinese Medicine and was performed according to the guidelines of the Helsinki Declaration. Written informed consent was obtained from all subjects.

\section{Consent for publication}

Not applicable.

\section{Competing interests}

The authors declare that they have no competing interests.

\section{Publisher's Note}

Springer Nature remains neutral with regard to jurisdictional claims in published maps and institutional affiliations.

\section{Author details \\ 'Shanghai Innovation Center of TCM Health Service, Shanghai University of Traditional Chinese Medicine, Shanghai 201203, China. ${ }^{2}$ School of Rehabilitation Science, Shanghai University of Traditional Chinese Medicine, Shanghai 201203, China. ${ }^{3}$ Zhangjiang Community Health Service Center of Pudong New District, Shanghai 201210, China. ${ }^{4}$ Institute of Digestive Diseases, China-Canada Center of Research for Digestive Diseases (ccCRDD), Longhua Hospital, Shanghai University of Traditional Chinese Medicine, Shanghai 200032, China.}

\section{Received: 12 July 2018 Accepted: 26 November 2018}

Published online: 20 December 2018

\section{References}

1. Alberti KG, Eckel RH, Grundy SM, Zimmet PZ, Cleeman II, Donato KA, Fruchart JC, James WP, Loria CM, Smith SC Jr. Harmonizing the metabolic syndrome: a joint interim statement of the international diabetes federation task force on epidemiology and prevention; National Heart, Lung, and Blood Institute; American Heart Association; world heart federation; international atherosclerosis society; and International Association for the Study of obesity. Circulation. 2009;120(16):1640-5.

2. Galassi A, Reynolds K, He J. Metabolic syndrome and risk of cardiovascular disease: a meta-analysis. Am J Med. 2006;119(10):812-9.

3. Ford ES, Li C, Sattar N. Metabolic syndrome and incident diabetes: current state of the evidence. Diabetes Care. 2008;31(9):1898-904.

4. Li R, Li W, Lun Z, Zhang H, Sun Z, Kanu JS, Qiu S, Cheng Y, Liu Y. Prevalence of metabolic syndrome in mainland China: a meta-analysis of published studies. BMC Public Health. 2016;16:296.

5. Li W, Song F, Wang X, Wang L, Wang D, Yin X, Cao S, Gong Y, Yue W, Yan $F$, et al. Prevalence of metabolic syndrome among middle-aged and elderly adults in China: current status and temporal trends. Ann Med. 2018;50(4):345-53

6. Wang F, Wu S, Song Y, Tang X, Marshall R, Liang M, Wu Y, Qin X, Chen D, Hu Y. Waist circumference, body mass index and waist to hip ratio for prediction of the metabolic syndrome in Chinese. Nutr Metab Cardiovasc Dis. 2009;19(8):542-7.
7. Yang $H$, Xin Z, Feng JP, Yang JK. Waist-to-height ratio is better than body mass index and waist circumference as a screening criterion for metabolic syndrome in Han Chinese adults. Medicine (Baltimore). 2017; 96(39):e8192.

8. Gasevic D, Frohlich J, Mancini GJ, Lear SA. Clinical usefulness of lipid ratios to identify men and women with metabolic syndrome: a cross-sectional study. Lipids Health Dis. 2014;13:159.

9. Chen BD, Yang YN, Ma YT, Pan S, He CH, Liu F, Ma X, Fu ZY, Li XM, Xie X, et al. Waist-to-height ratio and triglycerides/high-density lipoprotein cholesterol were the optimal predictors of metabolic syndrome in Uighur men and women in Xinjiang, China. Metab Syndr Relat Disord. 2015;13(5):214-20.

10. Li R, Li Q, Cui M, Yin Z, Li L, Zhong T, Huo Y, Xie P. Clinical surrogate markers for predicting metabolic syndrome in middle-aged and elderly Chinese. J Diabetes Investig. 2018;9(2):411-8.

11. Goldani H, Adami FS, Antunes MT, Rosa LH, Fassina P, Quevedo Grave MT, Morelo Dal Bosco S. Applicatility of the visceral adiposity index (VAl) in the prediction of the components of the metabolic syndrome in elderly. Nutr Hosp. 2015:32(4):1609-15.

12. Guo SX, Zhang XH, Zhang JY, He J, Yan YZ, Ma JL, Ma RL, Guo H, Mu LT, Li $S G$, et al. Visceral adiposity and anthropometric indicators as screening tools of metabolic syndrome among low income rural adults in Xinjiang. Sci Rep. 2016;6:36091.

13. Baumgartner RN, Heymsfield SB, Roche AF. Human body composition and the epidemiology of chronic disease. Obes Res. 1995;3(1):73-95.

14. Gu Z, Li D, He H, Wang J, Hu X, Zhang P, Hong Y, Liu B, Zhang L, Ji G. Body mass index, waist circumference, and waist-to-height ratio for prediction of multiple metabolic risk factors in Chinese elderly population. Sci Rep. 2018; $8(1): 385$.

15. Liu Y, Tong G, Tong W, Lu L, Qin X. Can body mass index, waist circumference, waist-hip ratio and waist-height ratio predict the presence of multiple metabolic risk factors in Chinese subjects? BMC Public Health. 2011;11:35

16. Kahn HS. The "lipid accumulation product" performs better than the body mass index for recognizing cardiovascular risk: a population-based comparison. BMC Cardiovasc Disord. 2005:5:26.

17. Amato MC, Giordano C, Galia M, Criscimanna A, Vitabile S, Midiri M, Galluzzo A. Visceral adiposity index: a reliable indicator of visceral fat function associated with cardiometabolic risk. Diabetes Care. 2010;33(4):920-2.

18. Bao Y, Lu J, Wang C, Yang M, Li H, Zhang X, Zhu J, Lu H, Jia W, Xiang K. Optimal waist circumference cutoffs for abdominal obesity in Chinese. Atherosclerosis. 2008;201(2):378-84.

19. Freedman DS, Jacobsen SJ, Barboriak JJ, Sobocinski KA, Anderson AJ, Kissebah AH, Sasse EA, Gruchow HW. Body fat distribution and male/female differences in lipids and lipoproteins. Circulation. 1990;81(5):1498-506.

20. Jiang B, Zheng Y, Chen Y, Li Q, Zhu C, Wang N, Han B, Zhai H, Lin D, Lu Y. Age and gender-specific distribution of metabolic syndrome components in East China: role of hypertriglyceridemia in the SPECT-China study. Lipids Health Dis. 2018;17(1):92.

21. DeLong ER, DeLong DM, Clarke-Pearson DL. Comparing the areas under two or more correlated receiver operating characteristic curves: a nonparametric approach. Biometrics. 1988:44(3):837-45.

22. Liu M, Wang J, Jiang B, Sun D, Wu L, Yang S, Wang Y, Li X, He Y. Increasing prevalence of metabolic syndrome in a Chinese elderly population: 20012010. PLoS One. 2013;8(6):e66233.

23. Taverna MJ, Martinez-Larrad MT, Frechtel GD, Serrano-Rios M. Lipid accumulation product: a powerful marker of metabolic syndrome in healthy population. Eur J Endocrinol. 2011;164(4):559-67.

24. Chiang JK, Koo M. Lipid accumulation product: a simple and accurate index for predicting metabolic syndrome in Taiwanese people aged 50 and over. BMC Cardiovasc Disord. 2012;12:78.

25. Tellechea ML, Aranguren F, Martinez-Larrad MT, Serrano-Rios M, Taverna MJ, Frechtel GD. Ability of lipid accumulation product to identify metabolic syndrome in healthy men from Buenos Aires. Diabetes Care. 2009;32(7):e85.

26. Zhang XH, Zhang M, He J, Yan YZ, Ma JL, Wang K, Ma RL, Guo H, Mu LT, Ding YS, et al. Comparison of anthropometric and Atherogenic indices as screening tools of metabolic syndrome in the Kazakh adult population in Xinjiang. Int J Environ Res Public Health. 2016;13(4):428.

27. Motamed N, Razmjou S, Hemmasi G, Maadi M, Zamani F. Lipid accumulation product and metabolic syndrome: a population-based study in northern Iran, Amol. J Endocrinol Investig. 2016;39(4):375-82. 
28. de Oliveira CC, Roriz AK, Ramos LB, Gomes Neto M. Indicators of adiposity predictors of metabolic syndrome in the elderly. Ann Nutr Metab. 2017; 70(1):9-15.

29. Ma CM, Lu N, Wang R, Liu XL, Lu Q, Yin FZ. Three novel obese indicators perform better in monitoring management of metabolic syndrome in type 2 diabetes. Sci Rep. 2017;7(1):9843.

30. Bozorgmanesh M, Hadaegh F, Azizi F. Diabetes prediction, lipid accumulation product, and adiposity measures; 6-year follow-up: Tehran lipid and glucose study. Lipids Health Dis. 2010;9:45.

31. Xia C, Li R, Zhang S, Gong L, Ren W, Wang Z, Li Q. Lipid accumulation product is a powerful index for recognizing insulin resistance in nondiabetic individuals. Eur J Clin Nutr. 2012;66(9):1035-8.

32. Dai H, Wang W, Chen R, Chen Z, Lu Y, Yuan H. Lipid accumulation product is a powerful tool to predict non-alcoholic fatty liver disease in Chinese adults. Nutr Metab (Lond). 2017;14:49.

33. Amato MC, Giordano C. Visceral adiposity index: an indicator of adipose tissue dysfunction. Int J Endocrinol. 2014;2014:730827.

34. Kouli GM, Panagiotakos DB, Kyrou I, Georgousopoulou EN, Chrysohoou C, Tsigos C, Tousoulis D, Pitsavos C. Visceral adiposity index and 10-year cardiovascular disease incidence: the ATTICA study. Nutr Metab Cardiovasc Dis. 2017;27(10):881-9.

35. Motamed N, Khonsari MR, Rabiee B, Ajdarkosh H, Hemasi GR, Sohrabi MR, Maadi M, Zamani F. Discriminatory ability of visceral adiposity index (VAl) in diagnosis of metabolic syndrome: a population based study. Exp Clin Endocrinol Diabetes. 2017;125(3):202-7.

36. Cordero A, Laclaustra M, Leon M, Casasnovas JA, Grima A, Luengo E, Ordonez B, Bergua C, Bes M, Pascual I, et al. Comparison of serum lipid values in subjects with and without the metabolic syndrome. Am J Cardiol. 2008;102(4):424-8.

37. Kawamoto R, Tabara Y, Kohara K, Miki T, Kusunoki T, Takayama S, Abe M, Katoh T, Ohtsuka N. Relationships between lipid profiles and metabolic syndrome, insulin resistance and serum high molecular adiponectin in Japanese community-dwelling adults. Lipids Health Dis. 2011;10:79.

Ready to submit your research? Choose BMC and benefit from:

- fast, convenient online submission

- thorough peer review by experienced researchers in your field

- rapid publication on acceptance

- support for research data, including large and complex data types

- gold Open Access which fosters wider collaboration and increased citations

- maximum visibility for your research: over $100 \mathrm{M}$ website views per year

At $\mathrm{BMC}$, research is always in progress.

Learn more biomedcentral.com/submissions 